\title{
The Introduction of a New Virtual Microscope into the eLearning Platform of the Medical University of Graz
}

\author{
http://dx.doi.org/10.3991/ijoe.v7i4.1785 \\ Herwig Rehatschek, Florian Hye \\ Medical University Graz, Graz, Austria
}

\begin{abstract}
In 2002 the Medical University of Graz (MUG) introduced its first Learning Management System (LMS). One of the first applications developed was a virtual microscope 1.0 (VM 1.0) to be used by students via a standard web browser. The basic idea was to offer the functionality of a microscope via an interactive application which is integrated within the LMS. The main advantages of that concept were saving costs, enhancing the flexibility for students and utilizing additional features and possibilities offered by new media. Cost saving was achieved by replacing hundreds of expensive physical microscopes by the virtual microscope, which can be used on any PC with a standard web browser. After more than 8 years the technology as well as the functionality of the VM 1.0 was now out-dated. We describe the stages to introduce a VM 2.0 within VMC/Moodle in order to help other universities who want to introduce a VM for their students to speed up the decision process and to avoid possible traps.
\end{abstract}

Index Terms-eLearning, virtual laboratory, new learning applications, multimedia applications

\section{INTRODUCTION AND BACKGROUND}

In 2002 the Medical University of Graz (MUG) introduced its first Learning Management System (LMS), the so called the Virtual Medical Campus Graz (VMC). Since then eLearning was fostered by the MUG and the VMC serves now as the portal to the eLearning content of the MUG [1], [2], [3], [4]. In 2002 also the virtual microscope 1.0 (VM 1.0) was developed and introduced for the students.

The basic idea was to offer the functionality of a microscope via an interactive application which is integrated within the LMS of the university. The main advantages of that concept were saving costs, enhancing the flexibility for students and utilizing additional features and possibilities offered by new media.

Due to the fact that having a virtual microscope thousands of students work with at the same time, we reduced the costs for our university, as there was no more the need to buy hundreds of expensive physical microscopes to be used in exercise lessons. Only one scanner device is needed to digitize the images of a microscope which can then be used by the virtual application. Since the virtual microscope is offered via the eLearning system flexibility for students in terms of time and location is provided as well. Students can use the virtual microscope whenever and wherever they want to. Last but not least we had the addi- tional possibility of setting markers in combination with explanatory text within the images to directly point out interesting regions for students within a digital sample.

In the meantime the concept of the virtual microscope has also been introduced broadly into the medical-IT sector. Huge commercial companies such as Aurora MSC [5] implemented complex and highly specialized virtual microscope applications to be used by medical experts like pathologists and dermatologists.

After more than eight years the technology as well as the functionality provided by our virtual microscope was now out-dated. With $1^{\text {st }}$ of October 2010 we introduced VMC/Moodle [6] as a new primary LMS at the MUG. In November 2010 we further decided to replace our existing VM 1.0 by a new software with state of the art technology and functionality. Within this paper we describe the stages defined in our concept to introduce a VM 2.0 within VMC/Moodle. Hence this paper shall help other universities who want to introduce a virtual microscope for their students to speed up the decision process and to avoid possible traps.

\section{EXISTING ViRTUAL MiCROSCOPE 1.0 AND REQUIREMENTS OF NEW VM}

\section{A. Virtual Microscope 1.0}

The existing virtual microscope consisted of three main components: the user interface for the students, a front end for authors to set markers and an upload client in combination with a server side process which calculated image pyramids out of the highest resolution image received (e.g. from a scanner). The user interface client connected to a server application which utilized a MS SQL 2000 database to manage the image pyramids. In Figure 1 the user interface for students is depicted.

In the upper area a mini map of the entire image was displayed, whereas next to the mini map navigation buttons and two buttons for zoom in and out could have been found. The red rectangle indicated the current magnified view which was shown in the lower area of the interface together with a scale (in this case 40 micro meter). The yellow arrow was a marker, which indicated a region that was important for the students (e.g. a certain kind of disease). All markers for a digital sample were provided by teachers or assistants and were listed at the lower left side of the interface. Students could have directly jumped to these regions just by clicking the link. 


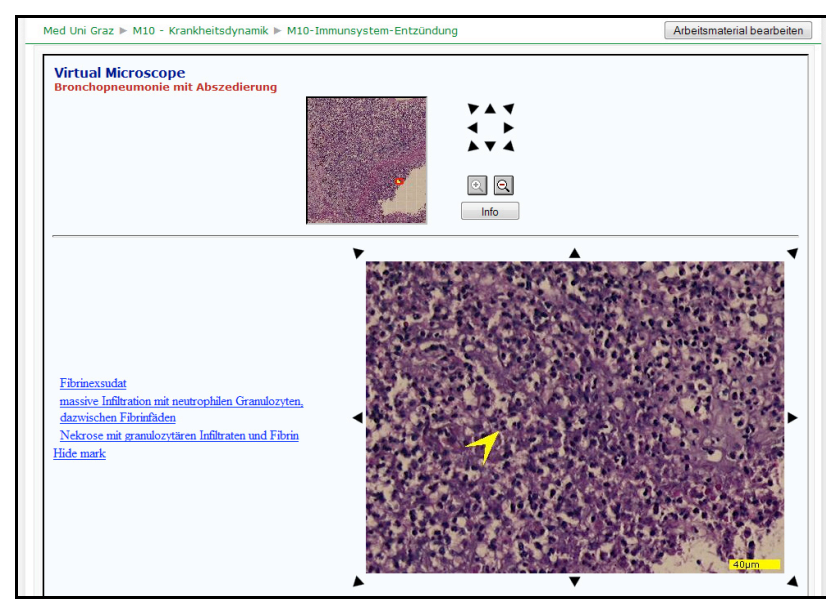

Figure 1. The virtual microscope 1.0 with mini map, navigation and markers

\section{B. User requirements on Virtual Microscope 2.0}

Together with our teachers we defined requirements for VM 2.0. The VM 2.0 has to provide the existing functionality of VM 1.0 and shall provide the following additional features:

Functionality

- Mini map and magnified view with at least 6 zoom levels, preferably progressive zoom shall be provided such as within Google maps [7]

- The zoom rectangle within the mini map can be moved simply by dragging the rectangle with the mouse, additionally navigation buttons such as in VM 1.0 shall be provided

- Possibility of setting of different markers (arrow, circle, rectangle, polygon) together with explanatory text

- Possibility of setting markers together with explanatory audio instead of text

\section{Technology}

- must be useable with standard web browsers, no installation of additional software, no stand alone clients $\rightarrow$ therefore the client should be implemented with latest Adobe Flash technology, which is already part of each common browser system (e.g. IE9, Firefox 3, Opera 11)

- if additional database software is necessary an open source database such as PostgreSQL or MySQL shall be applicable

User interface and navigation

From a user interface and navigational point of view, it was very important to keep the basic elements of VM 1.0, on the other hand it should provide a state of the art usability which is commonly known from today's web applications. There are four main areas of the VM which shall be clearly recognizable by the user and easily to use:

- navigation area with mini map

- information

- markers

- visualization window with object to investigate

In Figure 2 a mock-up of the desired user interface for the new VM is given.

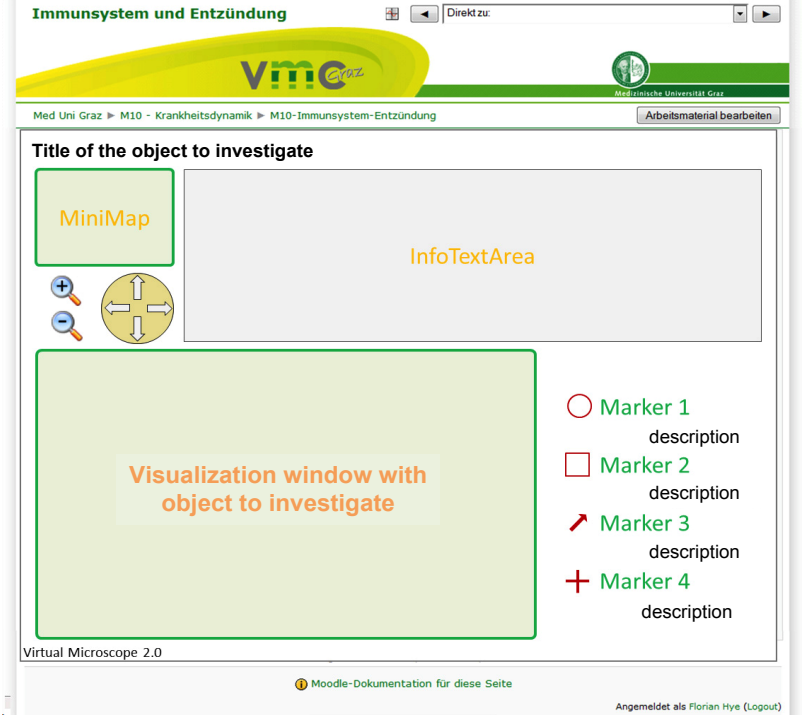

Figure 2. User interface mock-up for the new VM

In the upper left corner is the main navigation area consisting of a mini map, zoom and navigation buttons. The mini map is a small overview window of the object to be investigated and shall enable fast navigation by simply clicking into areas of interest. The main visualization window will then display a magnified view of the selected area. Ideally the part of the investigation object, displayed within the visualization window, is marked by a rectangle. This rectangle can be moved either directly with the mouse or by using the arrow buttons below the mini map. The zoom in and zoom out buttons allow to select the size of the rectangle and the part of the investigation object to be viewed.

The info text area shall hold information from the teacher in relation with the investigation object. Together with the markers it shall guide the student to areas of interest within the investigation object, e.g. areas of special interest within a tumor. Markers can be of different kinds of shapes indicating points of interest within the investigation object. These shapes should be arrows, rectangles, circles - depending on which kind of area the student shall concentrate on.

Last but not least the new VM must have a comfortable administration interface enabling teachers to annotate and prepare objects properly for the students, i.e. add markers and additional information.

\section{STATE OF THE ART}

Having the requirements defined we performed a market research. Basically there exist two groups of applications providing the functionality of a virtual microscope:

1. Desktop applications (client software has to be installed)

\section{Web based applications}

The larger group are the desktop applications, where also huge and highly specialized commercial solutions can be found. Since we had the requirement that the virtual microscope must be useable for students from any web browser, we continued investigation only within the second group. Here we found three applications which met our requirements: Zoomify [8], WebMic [9] and the VM of FH-Joanneum. 
PAPER

The Introduction of a New Virtual Microscope into the eLearning Platform of the Medical University of GraZ

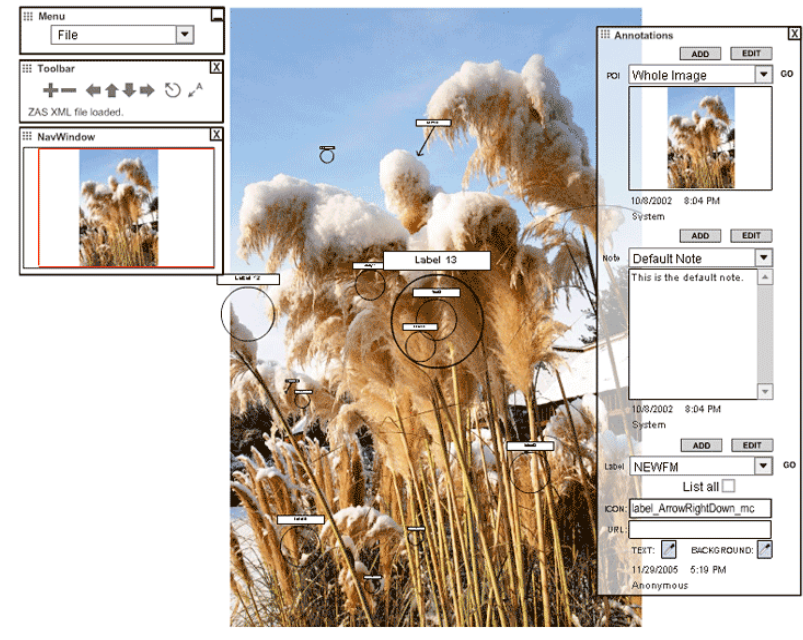

Figure 3. Example of the Zoomify application

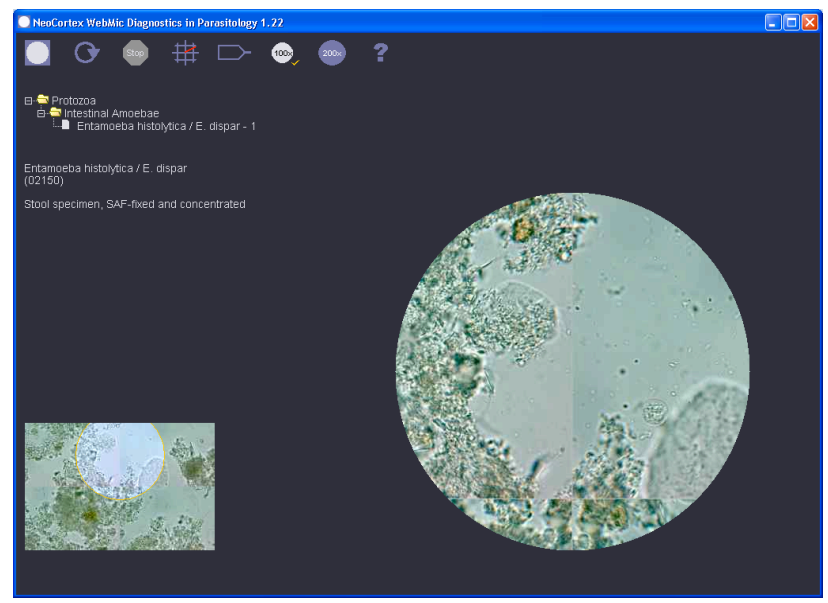

Figure 4. Example of a virtual microscope with WebMic

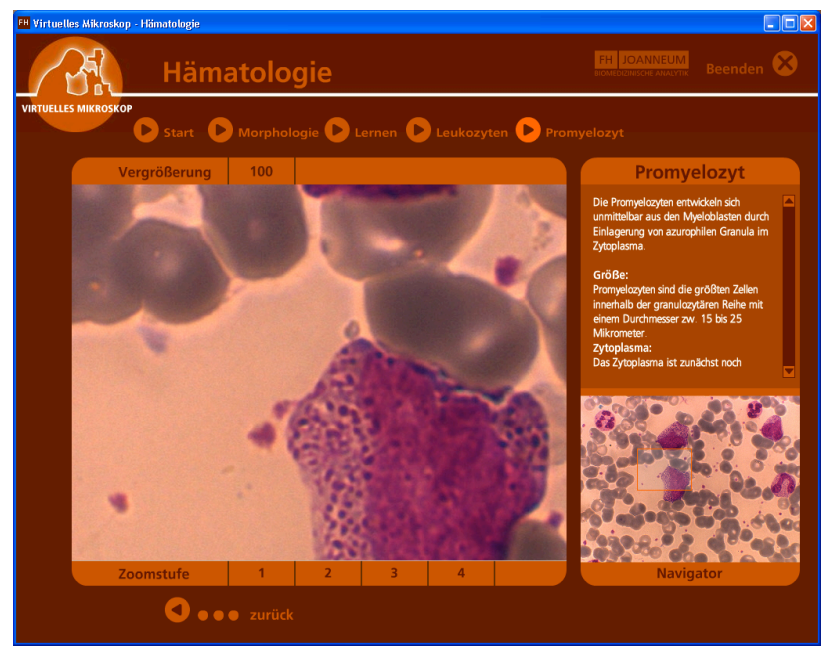

Figure 5. Example of the VM of the FH-Joanneum

\section{A. Zoomify}

Zoomify is a commercial solution of Zoomify Inc. The product in general supports zoom-and-pan of high resolution images for fast, interactive viewing on the web. In detail the product offers a number of special purpose applications of its viewer including aerial photos, product visualizations and medical microscopy. The company Zoomify exists for 11 years and has a large number of customers from the medical area including National Cancer Institute, die National Library of Medicine, University of Leeds und Virtual Dermatopology.

Good examples for medical applications can be found here [10], [11]. Technically Zoomify supports Flash technology for the client, the server application for importing visual objects is a $\mathrm{C}++$ executeable running under Windows and MacOS. Since January 2011 also a linux/unix version is available. Flash can be easily integrated within our LMS VMC/Moodle and also the offered functionality nearly perfectly matches our requirements. An example visualization with Zoomify is depicted in Figure 3

\section{B. WebMic}

WebMic was cordially developed by Anatomy Institute, Univ. Zürich, Dept. Cell Biology and Anatomy, Med. Univ. South Carolina, Charleston und NeoCortex. Examples for WebMic at NeoCortex can be found here [12], [13]. [13] is visualized in Figure 4.

It is based on Java technology and requires a java virtual machine to be installed at the client side, which makes it only semi optimal for our needs. WebMic supports an own quiz modus. The product is only available as a CDROM together with 159 investigation objects. We could not find a standalone version of this product, which makes it impossible to integrate it within our LMS VMC/Moodle.

\section{VM of the FH-Joanneum}

This virtual microscope was developed by the institute of biomedical analytics at the university of applied sciences FH-Joanneum in Graz, Austria [14]. The VM application currently holds objects for the areas of histology, haematology and cytology and supports a quiz tool for self evaluation of students. The VM is next to the students of the university also used by biomed Austria for staff training. An example of the VM of the FH-Joanneum is given below.

The client application is programmed in Flash and is very comfortable and supports also the grouping of several investigation objects to thematic groups. However, it does not support the set of markers within the investigation object. The VM of the FH-Joanneum is currently not commercially available but FH-Joanneum is open for cooperations and projects.

\section{COMPARISON AND PRODUCT DECISION}

Based on the market overview we evaluated the three applications according to the requirements defined by us. The results of this evaluation are given in Table I.

Based on this evaluation Zoomify meets best the requirements defined by the MUG on the virtual microscope 2.0. Due to its Flash technology this application can be easily integrated into our LMS VMC/Moodle. Even though a commercial product has the obvious disadvantage of generating a kind of dependency on the company, this is fairly compensated by the facts that the original source code is provided, the company has a huge number of customers (also in medical area) and a very attractive price is provided. 
TABLE I.

COMPARISON AND EVALUATION OF AVAILABLE WEB-BASED VIRTUALK MICROSCOPE APPLICARTIONS

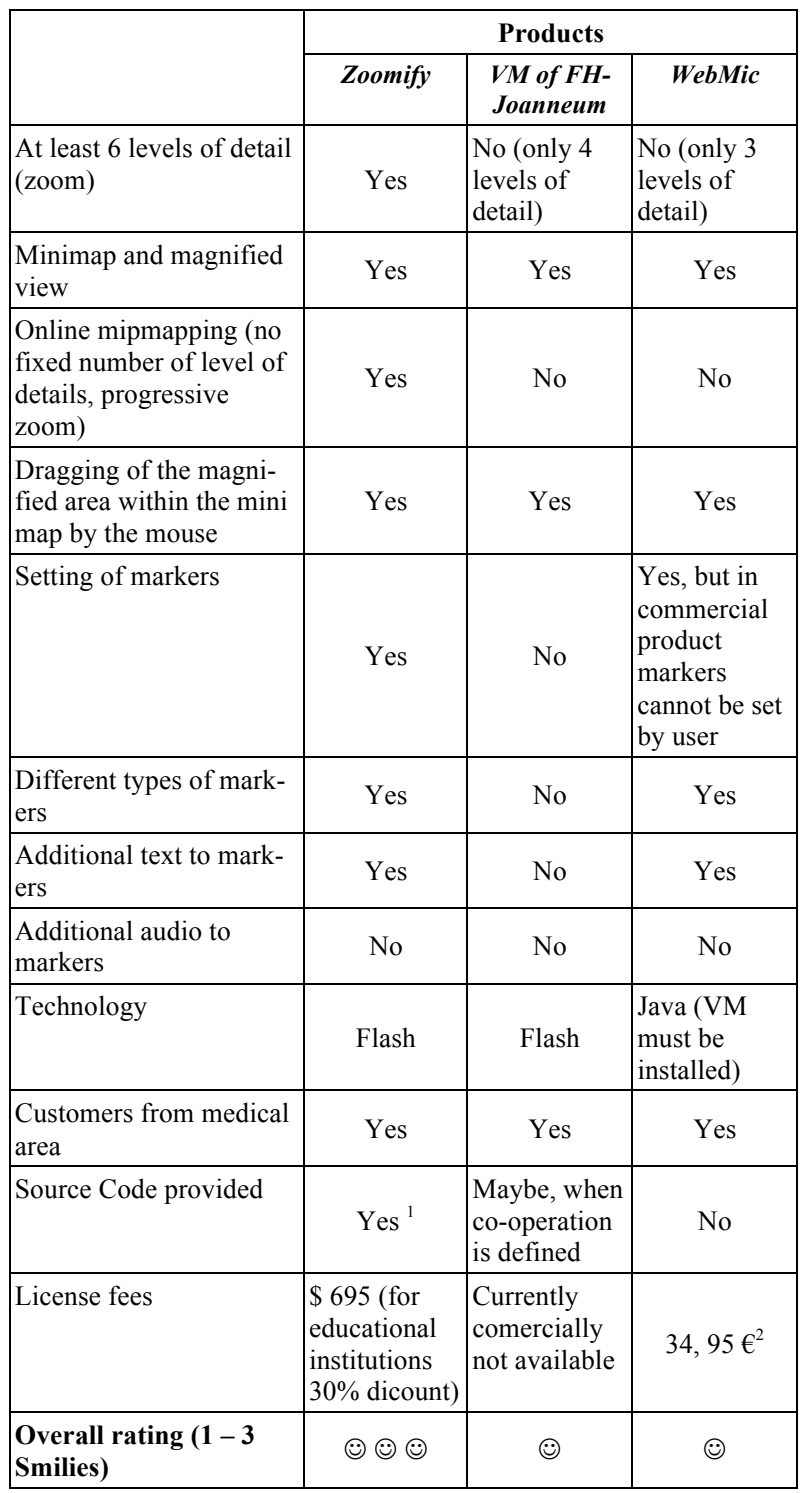

\section{TeChNiCAl REAlization AND MigRation}

With mid of February 2011 we had a concept for the introduction of the new virtual microscope based on Zoomify approved by the rector. In March 2011 we ordered the software and started with some initial field trials, in which we migrated a small number of old VM 1.0 samples and test the software within our LMS VMC/Moodle. Overall it can be stated that you shall have one local technician who is firm with Flash,XML and webserver technology. It took about one week to become familiar with the software; also it has to be mentioned that the support of Zoomify was very quick and constructive.

All these initial trials were very successful and the first migrated VMs were soon available within our LMS VMC/Moodle and used by the students. From begin of

\footnotetext{
${ }^{1}$ Partially. Source code available for client application. Upload client is written in $\mathrm{C}++$, no source code available.

${ }^{2}$ This is a CD-ROM application with a fixed data set. No really client /
} server web based application is commercially available.
May 2011 we started with the general migration process. All in all more than 350 old VMs will now be migrated to the new technology. Students are not hindered during this migration phase hence the migration can be done piece by piece. Each time a migration is finalized, the old VM is deleted and only the new VM is available.

During the migration process also the content quality is improved. For each migrated VM the corresponding teacher is asked to confirm the validity of this VM. In case the information is outdated the teacher provides either new information on the VM or the VM is deleted because it is no longer needed for the lecture.

For the migration of content to the new VM the investigated object in its highest resolution is needed. This image cannot be easily extracted from the old VM, because it only utilized image-tiles. In order to get the entire image all these tiles - in some cases 10.000 of tiles! - have to be gathered and sticked together. However, this is done only in case the teacher does not have the original object anymore. In this case we use the ImageJ utility offered by National Institute for Health [15] together with a script that puts all tiles together row by row. Since the memory requirements are quite high and the Java technology does not provide high performance we need this kind of migration is only the last resort. Usually teachers still have the high-res object or at least the original physical object. In the latter case we perform a new scan of the investigation object with our high-res Aperior image scanner and import the new object into our Zoomify VM.

In Figure 6 a VM with the new technology within our LMS VMC/Moodle is visualized.

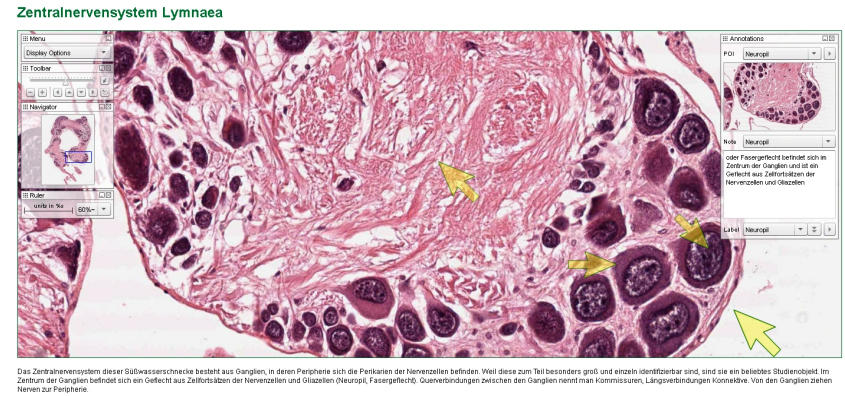

Figure 6. Example of a migrated VM within the MUG LMS VMC/Moodle

Within the upper left area the navigation functionalities are provided, including the mini map and an easy navigation within the mini map by moving the blue rectangle. In the upper right corner so called "points of interest" (POI) are provided in a drop down box next to explanatory text to this marker just below in the text box. These are areas of interest within the investigation object indicated by the teacher to be closely watched by the student. To each POI a marker is associated - in this example arrows - indicating exactly the position of the areas within the high resolution image to be closer investigated by the student. By clicking on the POI the main viewing window automatically positions itself to the corresponding marker. A magnification of the main area can be either achieved by using the mouse wheel, or by using the zoom tool with the navigation functions provided in the upper left corner.

Technically the markers and explanatory text for each VM are provided within an XML file which can be produced by a special client version of the VM (in fact the 
viewing application in edit mode) to be used only by authors. Authors will further need a local web server (e.g. IIS) and ASP in order to write XML files back to the server. Both, the image and the XML files are stored on a web server. The VM viewer itself is a Flash application and for each migrated object the application is integrated as a "link" within VMC/Moodle.

\section{EVALUATION RESUlts}

After the migration of about 20 old VMs to the new technology we performed an evaluation amongst the students. The evaluation consisted of 9 closed and one open question. For eight of the closed questions we used a scale from 1 to $6: 1=\mathrm{I}$ strongly agree, $2=$ very true, $3=$ is more like it, $4=$ does not apply to more, $5=$ makes little, $6=\mathrm{I}$ strongly disagree. For one closed question we used individual answers. For the open question we provided a text field. Technically the evaluation was performed within our LMS VMC/Moodle by utilizing the survey tool. We submitted the request to 267 students of Module $29\left(10^{\text {th }}\right.$ semester of human medicine) and 18 students from the $6^{\text {th }}$ study year $\left(11^{\text {th }}\right.$ and $12^{\text {th }}$ semester), hence 285 in total. We received 38 feedbacks, resulting in a return rate of $13.3 \%$. From the 38 feedbacks $61 \%$ came from male students, $39 \%$ from female students, $95 \%$ studied human medicine, $5 \%$ studied human and dental medicine.

Question \#1: "The usability of the new VM has significantly improved in comparison to the old VM". On a scale from 1 to $6(1=$ strongly agree $\ldots 6=$ strongly disagree $)$ $53 \%$ answered with $1,34 \%$ with 2 and $13 \%$ with 3 .

Question \#2: "I could immediately work efficiently with the new VM without having the need of a training". On a scale from 1 to $6(1=$ strongly agree .. $6=$ strongly disagree) $58 \%$ (!) answered with $1,37 \%$ with 2 , and $5 \%$ with a 3 .

Question \#3: "I prefer practices with the VM from "with options (a) at home with detailed additional information from the teacher, (b) in the classroom with mandatory presence (c) I do not care. 29\% answered with (a), $53 \%$ selected option (b) and 18\% option (c).

Question \#4: "The new VM technically offers all functionality in order to efficiently learn". On a scale from 1 to $6(1=$ strongly agree $\ldots 6=$ strongly disagree $) 26 \%$ answered with $1,29 \%$ with $2,39 \%$ with 3 and $5 \%$ with 5 .

Question \#5: "The new VM is stable and performs well". On a scale from 1 to $642 \%$ answered with $1,47 \%$ with 2 and $11 \%$ with 3 .

Question \#6: "I use the VM in module 29 for the preparation of the exam". On a scale from 1 to $645 \%$ answered with a $1,18 \%$ with a $2,18 \%$ with a $3,5 \%$ with a 4 and $14 \%$ with a 6.

Question \#7: "I use the VM mainly from" with options (a) a PC at home, (b) public PC provided by the MUG (c) Internet Café, (c) mobile device (d) other. 84\% selected option (a), $8 \%$ option (b) and $8 \%$ option (d).

These results are very encouraging. Possible interpretations and conclusions are discussed in the next chapter.

\section{CONCLUSIONS}

As preliminary conclusions we can state, that it is most probably more expensive to develop an own virtual microscope application than buying and adapting one of the already existing applications. For universities who want to introduce a virtual microscope for their students we strongly recommend to first define a detailed requirements document. It should be clearly defined, if a web based application or a local client application is needed. The latter has the advantage of more functionality and can be highly-specialized on the needs of researchers. However local clients have the disadvantage of having to install and continuously update software locally on all machines where the virtual microscope is used.

Then a market research shall be performed. There are many commercial but also open source virtual microscope applications with a vast number of features - hence it is very likely that an appropriate application will be found.

The integration of the new VM into our LMS VMC/Moodle works perfectly. VMs open in a separate window so the VM does not interfere with the content of the LMS. The visualization process works (zoom in/out, pan) very performant on clients we tested so far and can be compared to the speed of Google Maps. Also the stability of the application is very satisfying, we are not aware of any client application problems or crashes.

From the evaluation several important issues can be derived. Firstly, a very clear commitment of the students for the extensive usability improvement of the new VM was given. Also the tool seems to be very self explanatory no additional training is needed in order to work immediately efficient with the VM. Secondly it can be derived that a pure virtual offer of the virtual microscope is not preferred by the students. Also in connection with the open question it was stated that there should be at least one lecture in presence where the teacher explains the objects face to face and gives answers to potential questions. Finally the open question revealed wishes like the desire to print the images and notes, a strong wish for a presence lecture, and that the VM is very useful for the self evaluation of students. Finally from the open question testimonials like "ideally to learn", "successful update - congratulations", "good usability", "great service", "you did a great job! Now I will much more likely work with the VM than before" were very encouraging for us.

\section{REFERENCES}

[1] Staber R, Smolle J, Bauer P, Hye F, Thallinger S, Neges H, Reibnegger G.: „Virtueller Medizinischer Campus Graz: eine eLearning Umgebung wird 5 Jahre alt“. GMS Med Bibl Inf. 2007; 7(3):Doc43. ISSN 1865-066X, <URL (22 June 2011): http://www.egms.de/en/journals/mbi/2007-7/mbi000095.shtml >

[2] Smolle J, Staber R, Hye F, Jamer E, Macher S, Neges H et al.: "ELearning in the First SEMESTER of an Undergraduate Medical Curriculum.”, 2006. EDEN conference proceedings. Ref Type: Electronic Citation Ref ID: 9210, <URL (22 June 2011): http://www.eden-online.org/contents/conferences/submit/upload/ Smolle.doc $>$

[3] Smolle J, Staber R, Jamer E, Reibnegger G.: „Aufbau eines universitätsweiten Lern- Informationssystems parallel zur Entwicklung innovativer Curricula - zeitliche Entwicklung und Synergieeffekte“. In: Tavangarian D, Nölting K, editors. Auf zu neuen Ufern - E-Learning heute und morgen. Münster New York München Berlin: Waxmann, 2005: 217-226. Ref ID: 9019

[4] Smolle J, Staber R, Jamer E, Reibnegger G. Aufbau eines universitätsweiten Lern- Informationssystems parallel zur Entwicklung innovativer Curricula - zeitliche Entwicklung und Synergieeffekte. In: Tavangarian D, Nölting K, editors. Auf zu neuen Ufern - ELearning heute und morgen. Münster New York München Berlin: Waxmann, 2005: 217-226. Ref ID: 9019

[5] Aurora MSC, homepage, 2011. <URL (22 June 2011): http://www.auroramsc.com> 
PAPER

The Introduction of a New Virtual Microscope into the eLearning Platform of the Medical University of GraZ

[6] Moodle - Modular Object Oriented Dynamic Learning Environment, open source LMS, <URL (22 June 2011): http://www.moodle.org>

[7] Google Maps, homepage, 2011. <URL (22 June 2011): http://maps.google.at/>

[8] Zoomify, homepage, 2011. <URL (22 June 2011): http://zoomify.com>

[9] WebMic, Virtuelles Mikroskop, Allgemeine und Spezielle Histologie, Version 2.0, 2 CD-ROMs, EAN: 9783456834276. <URL (22 June 2011): http://www.buecher.de/shop/software/virtuellesmikroskop-allgemeine-und-spezielle-histologie-version-2-0-2-cdroms/anatomisches-institut-univ-z/products products/content/prod id/08740981/>

[10] Zoomify example, Institut für Anatomie und Zellbiologie and Institut für Biometrie, university of Saarland, Homburg. [URL (4 July 2011): http://www.mikroskopie-uds.de/]

[11] Zoomify example, Freie Universität Berlin. [URL (4 July 2011): http://www.vetmed.fu-berlin.de/einrichtungen/institute/we12/ images/galerie/endarteriitis/index.html]
[12] WebMic example \#1, NeoCortex. [URL (4 July 2011): http://130.60.57.53/webmic/allgspez/WebMicAllgSpez.html]

[13] WebMic example \#2, NeoCorex. [URL (4 July 2011): http://www.tropeduweb.ch/1.22WebMic_Parasitology_110805_w ww/parasitology/parasitology.html

[14] FH-Joanneum, homepage. [URL (4 July 2011): http://www.fhjoanneum.at]

[15] ImageJ, Image processing and analysis in Java, homepage. [URL (4 July 2011): http://rsbweb.nih.gov/ij/]

\section{AUTHORS}

Herwig Rehatschek and Florian Hye are with Virtual Medical Campus, Medical University Graz, Graz, Austria. \{Herwig.Rehatschek, Florian.Hye\}@medunigraz.at

This article is a modified version of a paper presented at the International Conference ICL2011, held in September 2011, in Piešt'any, Slovakia. 